\title{
A Novel Algorithm to Obtain Respiratory Rate from the PPG Signal
}

\author{
Mitali R. Ambekar \\ Postgraduate Student \\ Department of Electronics \\ FrCRCE Mumbai, India
}

\author{
Sapna Prabhu \\ Associate Professor \\ Department of Electronics \\ FrCRCE Mumbai, India
}

\begin{abstract}
Respiratory rate is a vital parameter which gives an indication of abnormal respiratory conditions. There are various methods which can be utilized to obtain breathing rate but they have certain drawbacks. In addition to $\mathrm{SpO}_{2}$ and heart rate measurement, PPG signal obtained from pulse oximeter can be used to get respiratory information which avoids use of additional sensor. In this paper, Ensemble Empirical Mode Decomposition algorithm has been proposed, which efficiently extracts respiratory information from PPG signal obtained by photo-plethysmography and decomposes a signal into IMF's while retaining features of the signal. This PPG signal has respiratory information embedded in it. It is observed that, this method helps in overcoming the drawbacks of traditional EMD method and giving 97\% average accuracy.
\end{abstract}

\section{General Terms}

Signal processing, Algorithms, motion artifacts.

\section{Keywords}

Respiratory rate; Photo-plethysmography; Beer- Lambert's law; PPG signal; EMD; EEMD

\section{INTRODUCTION}

The Human body consists of different physiological systems like the Cardiovascular System, Neuromuscular system, Excretory System, etc. Each system plays a vital role and communicates internally with each other and also with an external environment. Photo-plethysmography is a noninvasive technique, widely used to measure arterial oxygen saturation in blood. Signal processing techniques can be used to extract information regarding heart rate or breathing rate from photo-plethysmogram (PPG) which is a pulsatile waveform obtained using the Pulse Oximeter.

\subsection{Respiratory Rate}

Respiration is the process of exchanging gases during which living organisms take oxygen from the surroundings and give out carbon dioxide. It is an act of breathing. The normal volume of air inspired per breath is $500 \mathrm{ml}$ and while resting is about 5-8 liters of air per minute [1].

Vital signs are used to measure the body's basic functions which can give an indication of person's health as well as abnormal body conditions [2]. Respiratory rate is one of the most important vital signs. It is the primary indication of a person's respiratory illness. Respiratory assessment can identify changes happening in respiratory functions. Respiratory rate is the number of breaths taken by a person per minute and is measured when the person is at rest. It is also known as breathing rate, ventilation rate or breathing frequency. The normal ranges for a person's Respiratory Rate vary with age, weight, gender, and overall health. Respiratory Rate increases during Exercise, Fever, Asthma, Chronic Obstructive Pulmonary Conditions, Hyperventilation and Cardiac Arrest and decreases with the use of Narcotics, Alcohol as well as Abnormal Metabolic Conditions and Apnoea. Monitoring respiratory rate is thus useful to detect any form of respiratory changes. Typical methods used for measurement of respiratory rate are Nasal Thermistors, Displacement Method, Impedance Pneumography, $\mathrm{CO}_{2}$ Method, Spirometer [3]- [4], but these techniques increase patient discomfort during measurement. Hence photoplethysmography is used to extract respiratory activity.

Table No. 1 shows normal ranges of respiratory rate:

Table No.1 Normal Breathing Rates

\begin{tabular}{|l|c|}
\hline \multicolumn{1}{|c|}{ Individual } & $\begin{array}{l}\text { Respiratory Rate } \\
\text { (Breath Per Minute) }\end{array}$ \\
\hline New born & $30-60$ \\
\hline Infant (1 to 12 months) & $30-60$ \\
\hline Pre-schooler (3-5 years) & $22-34$ \\
\hline $\begin{array}{l}\text { School-age child (6-12 } \\
\text { years) }\end{array}$ & $18-30$ \\
\hline Adolescent (13-17 years) & $12-16$ \\
\hline Adult & $12-18$ \\
\hline
\end{tabular}

\subsection{Respiratory Pattern}

\section{Normal Breathing Pattern}

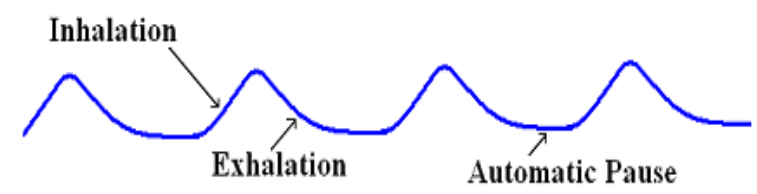

Fig 1: Normal respiratory pattern

Figure 1 shows the normal breathing pattern in which the rising edge of the pattern shows Inhalation and Falling Edge shows Exhalation. After exhalation there is an automatic pause which indicates no breathing is taking place. This portion is also known as the resting period [5]. The above pattern may change during abnormal respiratory conditions like Tachypnea, Bradypnea, Sleep Apnea, Hyperventilation and Hypoventilation. 


\subsection{Related Work}

PPG derived respiration is a topic of interest for many researchers. To obtain respiratory information from PPG signal, signal processing has to be done. Modified Multi Scale PCA (MMSPCA) method is combination of wavelet decomposition and principal component analysis (PCA), which modifies wavelet sub-band matrix using kurtosis and ECL (Energy contribution level) to remove Motion Artifacts (MA) efficiently. This paper concludes that the results obtained using MMSPCA method shows a higher degree of correlation with the respiratory signal [6]- [7]. ICA based DTCWT method is a combination of ICA (Independent Component Analysis) and DTCWT (Dual Tree Complex Wavelet Transform), which sufficiently extracts the respiratory information. It is observed that this method helps in reducing computational time as well as the computational cost [8]. Multi Parameter Respiratory Rate Extraction is a smart fusion method which segments respiratory induced amplitude, frequency and intensity variations. This method eliminates motion artifacts in the pulse segment and segments having low RR (Respiratory rate), but this elimination increases computational time [9]. Empirical mode decomposition (EMD) is a data analysis method which decomposes data into IMF's [10]- [11] and effectively reduces noise in the data. Zhaohua Wu and Norden E. Huang suggest Noise-Assisted Data Analysis (NADA) i.e. Ensemble Empirical Mode Decomposition (EEMD) [12], which uses properties of white noise to analyse the climate data and the voice data. This paper concludes that the results obtained using EEMD shows a drastic improvement, uniqueness and robustness over other techniques.

\section{PLETHYSMOGRAPHY}

Photo-plethysmography is a method which makes use of light energy and determines changes in the blood volume. In this technique, a light source and light sensor are placed on the opposite or on the same side of peripheral body parts such as finger, ear lobe, or toe, for measuring the alterations in light intensity that passes through the tissue. The result obtained from photo-plethysmography is known as a photoplethysmogram (PPG). It is a low cost, simple, portable, and non-invasive technology which usually used for measuring oxygen saturation, blood pressure, heart rate and pulse rate.

The attenuation of light is mainly caused by changes in the blood volume and in the orientation of blood vessels, and blood vessel wall movement. A typical PPG signal comprises of two main components: AC and DC [2]. AC is the pulsatile component as detected by the light sensor, pertaining to the synchronous changes in the blood volume with each heartbeat. This AC component is superimposed onto a large DC component that relates to the tissues and to the average blood volume. The DC component must be removed to measure the $\mathrm{AC}$ waveform with a high signal-to-noise ratio, since the useful AC signal is only a very small portion of the whole signal [3]- [7]. The PPG signal also contains the respiratory-induced intensity variations (RIIV). RIIV occur because of the changes in the intra-thoracic pressure with an inspiration and expiration affecting the peripheral venous return to heart, per respiration cycle. Figure 2 depicts $\mathrm{AC}$ and DC components of PPG signal [13] and figure 3 shows typical PPG signal [14]

A Pulse Oximeter is an instrument which indicates the changes in the absorption of light in the tissue by detecting changes in light transmission through the vascular bed. This absorption of a light is based on Beer- Lambert's law [2]- [4]: It states that If a monochromatic light of intensity to enters a medium, part of this light is transmitted through the medium and the rest is absorbed.

$$
\mathbf{I}=\mathbf{I}^{-\mathbf{k C b}}
$$

The law suggests that there is a linear relationship between the absorbance and concentration of the absorbing material. The light sources are consists of red LED (660nm) and infrared LED $(940 \mathrm{~nm})$ and thus, the level of changes detected reflects the change in absorption of light due to the tissue.

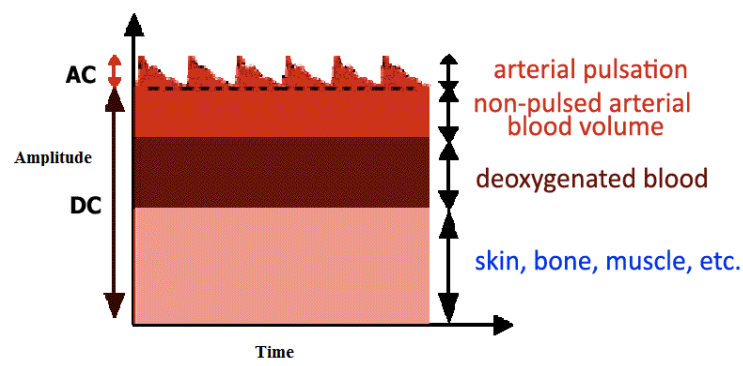

Fig 2: AC and DC components

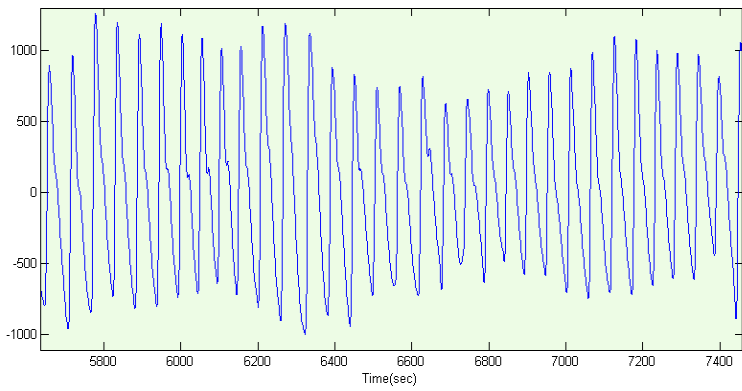

Fig 3: Typical PPG Signal

\section{EEMD}

Empirical mode decomposition (EMD) is a technique which is widely used for processing non-stationary, non- periodic signals, it still has certain difficulties. One of the drawbacks of EMD is frequent appearance of mode mixing. i.e. EMD cannot identify IMF's whose frequencies are very close to each other. Upper and lower envelope formation is an essential step in IMF extraction process, but this stage has serious disadvantage that it shows undershoot and overshoot problems. This end effects need improvement.

To overcome the difficulties in EMD, noise- assisted data analysis (NADA) [12] method is presented. In this paper, EEMD algorithm is used to find respiratory rate from the PPG signal. Ensemble Empirical Mode Decomposition is a method which produces IMF's having ensemble mean of signal and white noise. This method uses statistical properties of white noise and showed that EMD acts like a dyadic filter band when applied to white noise. With this ensemble approach, this method can clearly separate the scale naturally without any a priori subjective criterion selection as well as can reduce signal intermittency.

In EEMD, white noise is added with the signal to be processed. This added white noise populates the whole timefrequency space uniformly. The signal is added to this white background, the bits of signal of different scale are projected onto proper scale defined by the white noise background [12]. To such signals when EEMD is applied, noisy results are 
produced. During decomposition noise is cancelled in ensemble trials and meaningful IMF's are obtained. EEMD uses scale separation property of EMD. By adding white noise EEMD eliminates the problem of mode mixing. The ensemble mean is treated as the true answer, for, in the end, the only persistent part is the signal as more and more trials are added in the ensemble [12].

The added white noise is treated as random noise. Although adding noise may result in smaller signal to- noise ratio, the added white noise will provide a uniform reference scale distribution to facilitate EMD; therefore, the low signal-noise ratio does not affect the decomposition method but actually enhances it to avoid the mode mixing [12].

Steps for EEMD are as follows:

1. Add white noise to signal.

2. Decompose noise added signal into IMF's.

3. Repeat above step 1 and 2 if signal does not satisfy conditions of IMF's.

4. Obtain the magnitude spectrum for each IMF

5. Identify IMF falling in frequency range of $0.2 \mathrm{~Hz}-$ $0.33 \mathrm{~Hz}$.

6. Calculate the ensemble mean of IMF as a final result.

\section{RESULTS AND DISCUSSION}

Records of the PPG signals are obtained from MIMIC database in the Physiobank ATM. Records are identified suitable for proposed algorithm for the extraction of breathing rate. Records contain PPG signal with simultaneously recorded respiratory signal. This respiratory signal can be used as a reference signal. Signals in the records are recorded using sampling frequency of $125 \mathrm{~Hz}$ [15]. Neat portions of recorded data are taken having no missing data. All the records are processed using signal processing toolbox of MATLAB R2013b. Experimental results obtained using proposed method is as follows:

A neat portion of Record\# $055 \mathrm{~m}$ having duration of one minute is selected. Figure 4 shows the original PPG signal and its magnitude spectrum is depicted in Figure 5. Figure shows that highest frequency peak is related to cardiac frequency and respiratory peak lies between frequency ranges of $0 \mathrm{~Hz}-5 \mathrm{~Hz}$.

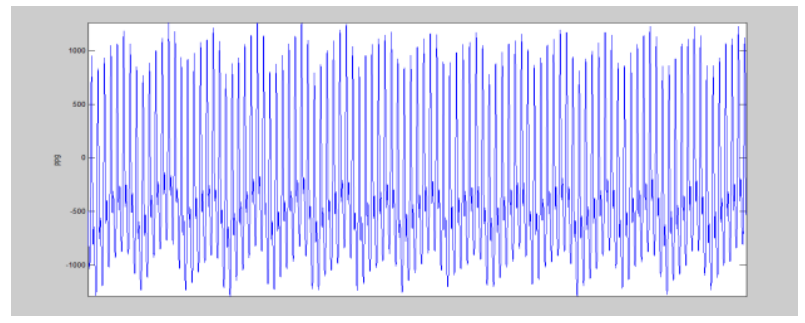

Fig 4: Original PPG Signal of Record\# 055m

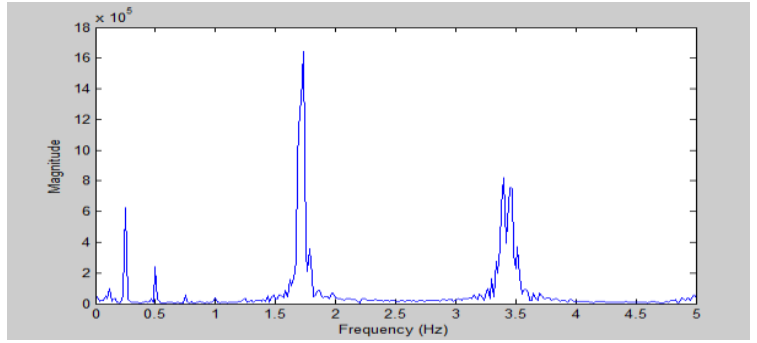

Fig 5: Magnitude Spectrum of Original PPG Signal
Figure 6 shows the extracted IMFs using EEMD algorithm. IMF extraction is done to suppress the cardiac information.

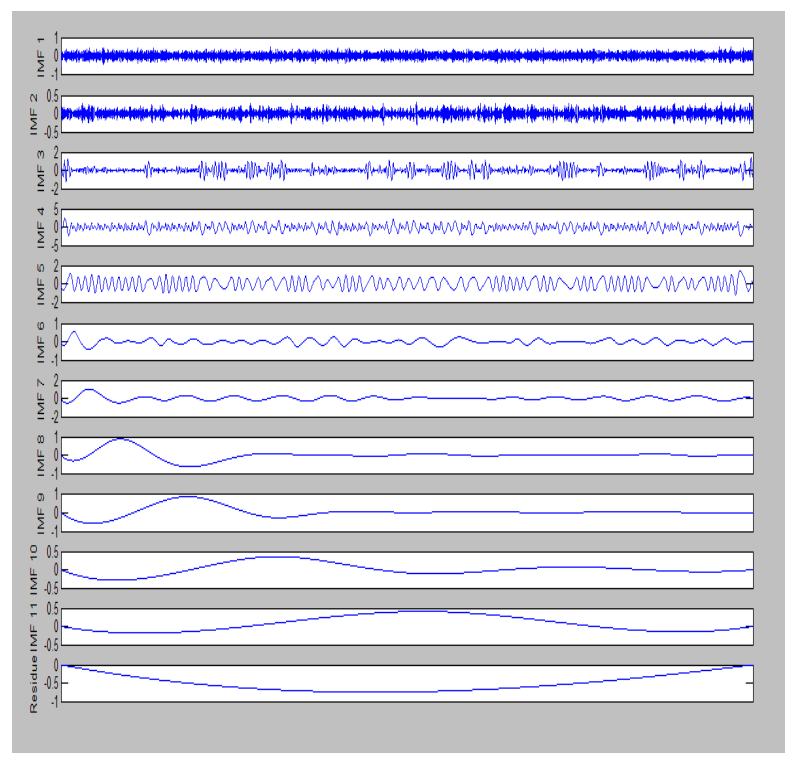

Fig 6: Extracted IMFs of Record \#055m

From the obtained IMFs, the IMFs falling in a frequency range of $0.2 \mathrm{~Hz}$ to $0.33 \mathrm{~Hz}$ were selected and added to obtain surrogate respiratory signal. Figure 7 shows the magnitude spectrum of a surrogate respiratory signal which shows that peaks related to cardiac frequencies in PPG signal are suppressed during signal processing and only respiratory peak is enhanced

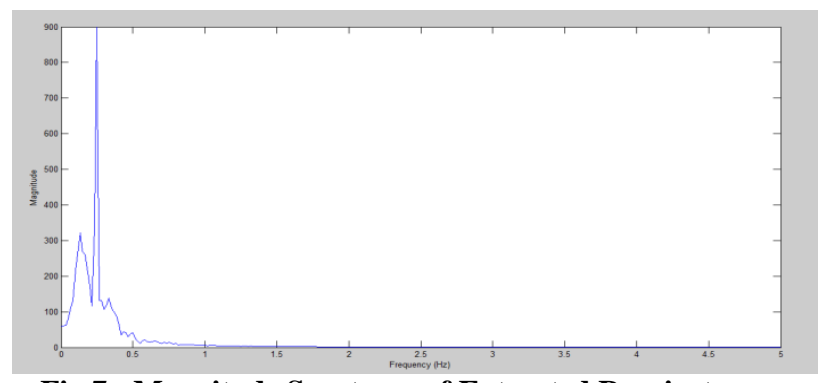

Fig 7: Magnitude Spectrum of Extracted Respiratory Signal

Some records from MIMIC database are tested using EEMD algorithm. This algorithm is applied to signals of one-minute duration and the obtained original and estimated respiratory rates are listed in following table:

Table No.3 Results by EEMD

\begin{tabular}{|c|c|c|c|c|c|}
\hline Record\# & ORR & ERR & AR & RCC & MSC \\
\hline $041 \mathrm{~m}$ & 14 & 13.98 & 99.86 & 0.99 & 0.86 \\
\hline $055 \mathrm{~m}$ & 15 & 15 & 100 & 1 & 0.87 \\
\hline $240 \mathrm{~m}$ & 15 & 15 & 100 & 1 & 0.77 \\
\hline $252 \mathrm{~m}$ & 15 & 13.99 & 93.27 & 0.87 & 0.66 \\
\hline $284 \mathrm{~m}$ & 15 & 13.99 & 93.27 & 0.90 & 0.67 \\
\hline $408 \mathrm{~m}$ & 15 & 15 & 100 & 1 & 0.72 \\
\hline $472 \mathrm{~m}$ & 18 & 18 & 100 & 1 & 0.72 \\
\hline
\end{tabular}

ORR- Original respiratory rate, ERR- Estimated respiratory rate, AR- Accuracy rate, RCC- Relative correlation coefficient, MSC- Magnitude Squared Coherence 
A match between derived respiratory signal and original respiratory signal can be obtained by calculating degree of similarity in time domain in terms of relative correlation coefficient (RCC). A degree of similarity in frequency domain can be obtained in terms of magnitude squared coherence (MSC). The accuracy rate is a ratio of estimated respiratory rate to the original respiratory rate.

\section{CONCLUSION}

PPG signal is a non- stationary signal and also it is very noisy due to motion artifacts. Hence in this paper, EEMD method is proposed to obtain necessary respiratory information from PPG signal. This algorithm is suitable for processing nonstationary signals. This paper shows significant improvement in results obtained by EEMD over EMD. EEMD method is efficiently extracting the respiratory information from the PPG signal and also separating IMF's without mode mixing. The average accuracy obtained by presented method is $97 \%$, thus obtained results are showing the efficiency of EEMD. The algorithm has been implemented in MATLAB R2013b.

Future work shall include feature extraction from PPG signals which can be used for identification of abnormal respiratory conditions.

\section{ACKNOWLEDGMENTS}

We would like to express our sincere thankful appreciation to Principal (FrCRCE), Dr. Srija Unnikrishanan and Head of Department (Electronics, FrCRCE), Dr. Deepak V. Bhoir, for their encouragement, advice, and support in the preparation of this paper.

\section{REFERENCES}

[1] D. J. Meredith, D. Clifton, P. Charlton, J. Brooks, C. W. Pugh and L. Tarassenko, "Photoplethysmographic derivation of respiratory rate: a review of relevant physiology", Journal of Medical Engineering \& Technology, 2012; 1-7, Early Online.

[2] Paul D. Mannheimer, "The Light-Tissue Interaction of Pulse Oximetry”, Vol. 105, No. 6, December 2007

[3] R.S. Khandpur, Handbook of Biomedical Instrumentation, 2nd ed. New Delhi: Tata McGraw- Hill, 2010

[4] J. G. Webster, Medical Instrumentation: Application and Design, 3rd ed. New York: Wiley, 2007

[5] http://www.normalbreathing.com/index-rate.php

[6] K. V. Madhav, M. R. Ram, E. H. Krishna, K. N. Reddy, and K. A. Reddy,"Use of multi scale PCA for extraction of respiratory activity from photoplethysmographic signals," in Proc. 29th IEEE I2MTC, Graz, Austria, May 13-16, 2012, pp. 1784-1787.

[7] K. Venu Madhav, Raghu Ram, Hari Krishna, Nagarjuna Reddy Komalla, and K. Ashoka Reddy, "Robust Extraction of Respiratory Activity From PPG Signals Using Modified MSPCA" IEEE TRANSACTIONS ON INSTRUMENTATION AND MEASUREMENT, VOL. 62, NO. 5, MAY 2013

[8] M. Raghu Ram, K. Venu Madhav, Ette Hari Krishna, Nagarjuna Reddy Komalla, Kosaraju Sivani, and K. Ashoka Reddy, "ICA-Based Improved DTCWT Technique for MAReduction in PPG Signals With Restored Respiratory Information", IEEE TRANSACTIONS ON INSTRUMENTATION AND MEASUREMENT, VOL. 62, NO. 10, OCTOBER 2013

[9] Walter Karlen, Srinivas Raman, J. Mark Ansermino, and Guy A. Dumont, "Multiparameter Respiratory Rate Estimation from the Photoplethysmogram", IEEE TRANSACTIONS ON BIOMEDICAL ENGINEERING, VOL. 60, NO. 7, JULY 2013.

[10] K. V. Madhav, M. R. Ram, E. H. Krishna, K. N. Reddy, and K. A. Reddy, "Estimation of respiratory rate from ECG, BP and PPG signals using empirical mode decomposition," in Proc. 28th IEEE I2MTC, Hangzhou, China, May 10-12, 2011, pp. 1611-1664

[11] N.E. Huang, Z. Shen, S.R. Long, M.L.Wu, H.H. Shih, Q. Zheng, N.C.Yen, C.C.Tung and H.H. Liu, "The empirical mode decomposition and Hilbert spectrum for nonlinear and non-stationary time series analysis," Proc. Roy. Soc. London A, Vol. 454, pp. 903-995, 1998.

[12] ZhaohuaWu and Norden E. Huang, "Ensemble Empirical Mode Decomposition: A Noise Assisted Data Analysis Method", Advances in Adaptive Data Analysis, vol. 1, no. 1, pp. 1-41, 2009.

[13] http://www.angioscan.ru/en/radiascan/devices/radiascan501/16 science/publish

[14] http://www.snipview.com/q/Monitoring_\%28medicine\% 29

[15] http://www.physionet.org/physiobank/database/mimicdb/ availableat:Indexof/physiobank/database/mimicdb/037. 